\title{
Experiences, Challenges and Lessons from Rolling Out a Rural WiFi Mesh Network
}

\author{
Carlos Rey-Moreno \\ Computer Science \\ University of Western Cape \\ Bellville 7535, South Africa \\ crey-moreno@uwc.ac.za

Zukile Roro
Computer Science
University of Western Cape
Bellville 7535, South Africa
2708776@uwc.ac.za

\author{
William D. Tucker \\ Computer Science \\ University of Western Cape \\ Bellville 7535, South Africa \\ btucker@uwc.ac.za \\ Masbulele Jay Siya \\ TransCape \\ Mankosi Community \\ Ngqeleni 5140, South Africa \\ jaysiya26@gmail.com
}

\author{
Nicola J. Bidwell \\ CSIR-Meraka, Pretoria and \\ NMMU, Port Elizabeth \\ South Africa \\ nic.bidwell@gmail.com \\ Javier Simo-Reigadas \\ Signal and Communications \\ Rey Juan Carlos University \\ Fuenlabrada 28943, Spain \\ jsimo@urjc.es
}

\begin{abstract}
The computing for development community knows that technology interventions involve consideration of social, technical and environmental factors. Research into WiFi solutions has fallen off as ubiquitous mobile solutions penetrate even the deepest rural communities worldwide. This paper argues that the latest wave of WiFi mesh networks offers benefits that traditional top-down $\mathrm{WiFi}$ and mobile networks do not. In addition, we propose ethnographic and participatory methods to aid the effective rollout of mesh inverse infrastructure with and for a given community. This paper describes and then analyzes a mesh for voice rollout within a situated context. We explain how to conduct informed community co-design and how to factor in local socio-political concerns that can impact on the design, rollout and subsequent maintenance of community-based wireless mesh networks. While we have not yet analyzed baseline and initial usage data, we do have new lessons to offer.
\end{abstract}

\section{Categories and Subject Descriptors}

C.2.1 [Network Architecture and Design]: Network Communications/Network Topology/Wireless Communication; C.2.2 [Network Protocols]: Routing Protocols/Wireless Mesh Networks

\section{General Terms}

Design, Human Factors, Telecommunications, Baseline study

\section{Keywords}

Community co-design, Participatory and Ethnographic Methods, VoIP, Inverse Infrastructure

Permission to make digital or hard copies of all or part of this work for personal or classroom use is granted without fee provided that copies are not made or distributed for profit or commercial advantage and that copies bear this notice and the full citation on the first page. To copy otherwise, to republish, to post on servers or to redistribute to lists, requires prior specific permission and/or a fee.

DEV'13, Jan 11-12, 2013, Bangalore, India.

Copyright 2013 ACM 978-1-4503-1856-3/13/01 ...\$15.00.

\section{INTRODUCTION}

WiFi networks have long been proposed to improve connectivity in rural areas of developing countries based on their low-cost, the widespread availability of compatible devices, and the use of non-licensed bands $[18,6]$. There are several notable initiatives in the literature and in practice that took those propositions seriously. Over the years, these projects have demonstrated feasibility and sustainability, and many continue to provide the same services initially envisioned, if not more, for low cost wireless networks in disadvantaged areas around the world $[17,22,1]$. As technical challenges of low cost WiFi were being overcome by such initiatives, incumbent telcos worldwide achieved wide $2 \mathrm{G}$ coverage for the provision of voice services and narrow-band data. Concomitant roll-out of $3 \mathrm{G}$ in many countries now provides resilient and robust services, all based on Internet Protocol (IP). The massive $2 \mathrm{G}$ and $3 \mathrm{G}$ coverage has perhaps led to a decrease in interest from the research community to push the limits of WiFi infrastructure and services to remote and rural scenarios, due to the limitations of WiFi in such areas.

Ironically, the wide availability of mobile phones and their services can actually be problematic for the most impoverished. Mobile phones services are homogeneously priced for users regardless of their location or income level. Thus even though users may adjust their mobile use according to cost, ubiquitous mobile services can remain unaffordable, and therefore economically inaccessible, for many segments of the population in developing countries, especially in rural areas $[19,10]$. Telcos tailor offerings to clients able to provide the most average revenue per user (ARPU), and costs for the impoverished can be prohibitive for basic voice, texting (SMS) and data. Thus, even ubiquitous physical availability does not necessarily translate to ubiquitous accessibility. The so-called 'digital divide' remains despite the world's poor being immersed in the invisible ether comprising ubiquitous $2 \mathrm{G}, 3 \mathrm{G}$ and beyond. Alternative technologies such as WiMAX seem be too expensive and inappropriate for rural deployment [26]. OpenBTS and related technologies also offer low cost wireless solutions [2], although they attract the attendant issues of licensed spectrum policy. TV white spaces (TVSW) may offer a more viable option, although it is currently on the outer horizon of wireless re- 
search and implementation. This leaves low cost WiFi and WiFi-like technologies, and their services, as technologies that can still offer an attractive bridge over gaps caused by the expense of cellular infrastructure, devices, and services.

The deployment of $\mathrm{WiFi}$ in rural areas, even using long distance links, is well understood. The many cookbooks, e.g. [15], and companies, e.g. Mikrotik and Ubiquiti, have together enabled the spread of easier and cheaper WiFi deployment; more than it ever was in recent years. Most of these solutions are based on using WiFi in infrastructure mode, comprising routers with multiple radio cards creating different and independent WiFi networks at each hop. This approach has been proven robust enough to provide the services desired [29]. Ad hoc, or mesh mode, has been overlooked or disparaged as non-scalable or ineffective at long distances $[8,29]$. This is contradictory with recent developments and larger scale deployments, e.g. FreiFunk and the Village Telco community networks, and suggests that WiFi mesh networks have actually not been sufficiently explored.

WiFi-based mesh networks in urban areas of both developed and developing countries are spreading quickly [16]. The lessons learned from these initiatives have enabled the existence of 'off-the-shelf' mesh hardware and software that ease the deployment of wireless mesh networks, e.g. the Mesh Potato of the Village Telco [32]. Many of these mesh 'kits' make extensive use of open source software, e.g. mesh routing protocols, and the accompanying active support from an associated online community. There are already several significant examples of a wireless mesh network providing services to end-users that pay for them [28], of which very few exist in rural areas of developing countries [21].

Notable initiatives that utilized WiFi revealed that the existence of a technology alone does not guarantee sustained success in the deployment, even for constrained scenarios [11]. Rather, success must be understood in terms of long term sustainability of the goals of the network and their impact. Such findings resonate with those of the monitoring and evaluation community [13]. Technology developers and evaluators alike appear to agree that social, political and cultural factors must be considered alongside purely technical and environmental considerations.

This paper describes the challenges encountered and the lessons learned while rolling out a wireless mesh network with and for a rural impoverished community in order to establish a baseline study for an ongoing effort to measure the impact and sustainability of rural mesh-enabled services. Almost a decade of experience in and around this community has lead us to employ what we consider to be a more socially aware approach to the process of rolling out a rural wireless mesh network. At this time, the initial mesh network has only just been installed, and we are still in the process of evaluating questionnaire data collected within the community. However, the process of establishing this baseline study revealed that the non-technical guidelines offered in the literature for deploying WiFi in rural areas of developing countries are not necessarily appropriate for wireless mesh networks, as they are entirely based on inverse infrastructure, as opposed to top-down [33]. We have identified additional concerns that need to be addressed, and would like to share them with the computing for development (DEV) community. While some authors [11, 20, 30] mention the pertinence of cultural factors, very little is said about how to approach them.
This paper addresses these concerns, especially with respect to the influence of intra-community politics on the design and rollout phase that we have encountered during the early stages of this mesh project, with the hope that others can benefit from our socially aware approach and the lessons we have learned. We will often stress the situated locality of our particular project. This may lead readers to the conclusion that it is difficult to generalize our experience and the derived conclusions to different contexts and cultures. Nevertheless, we strongly believe the conclusions presented herein could serve similar projects elsewhere. Even if not deploying similar infrastructure and services, e.g. introducing DEV into a rural area, we feel that our experience rolling out the baseline for this mesh network highlighted a number of significant issues that can at least help the reader to become more aware of the existence of similar issues in another locality; factors that could influence the achievability and sustainability of 'real access and real impact' [13].

The paper is structured as follows. The next section provides background information regarding the context where the initiative is located. A methodology section describes the steps taken to design and rollout the initial mesh network, organized by essential components of our socially aware approach. The next section discusses the new lessons we learned while rolling out the network, mostly informed by an ethnographic analysis of how social aspects impinge upon technical ones. A final section concludes the paper and outlines future work.

\section{CONTEXT}

Mankosi community belongs to a 'ward', and is one of the 31 wards that comprise the a rural 'municipality', in the Eastern Cape province of South Africa. The ward is the smallest administrative division in the country, and is represented by a warden who is elected democratically by the ward's population. It is difficult to disaggregate population data at the ward level, since such data is aggregated at the municipal level. According to national sources, Nyandeni municipality has a population of over 320,000 people, $99.6 \%$ of which belong to an ethnicity and culture defined by the use of a singular spoken language, isiXhosa. This particular municipality has one of the lowest Human Development Index (HDI) values in the province, 0.34; far below both the provincial and national averages of 0.48 and 0.55 , respectively.

Residents often use the word 'community' to describe those living within a 'location' and governed by a local Tribal Authority (TA). Sometimes a community can belong to two different wards simultaneously because a ward is a governmental division and a community is not. In each community, land is organized according to traditional culture, as has been done for many generations. This process is governed by the TA, a traditional political institution inherited patrilineally and parallel to legislative and political institutions existing in more urbanized areas. The TA comprises a Headman and a number of Sub-headmen, one from each village composing the community, each of whose homestead is also a site for administration.

Mankosi community is composed of approximately 580 households, based on data collected directly from the field, with each household composed of up to five adults and seven children, scattered into twelve villages of different sizes. These households are geographically scattered across about $30 \mathrm{~km}^{2}$ 
of very hilly and grassy land, scored by rivers and smaller water pathways, and dotted by the remaining clusters of indigenous forest and a generous sprinkling of grazing cattle. Very few passable dirt roads penetrate the area, although those that do are drivable in most weather conditions with $4 \times 4$, except when the bridges flood. Housing in the community is primarily a traditional thatched roof 'rondavel' with mud-brick walls. Small family clusters of rondavels are often co-located alongside the less numerous, yet conspicuously constructed, rectangular tin-roofed houses. These are regarded as a symbol of prosperity and also more easily allow for caching water into large plastic tanks. Collected rain water is usually not enough for a family, especially in the dry season, and water needs to be collected from water points installed by the government. Women typically walk up to several kilometers with 20 liters of water on their heads. Very often, the few well-educated people emigrate to find work in a more urban area. Thus income is sent home, and for local residents comes mainly from governmental pension payments. Income can also come from land-use, mostly subsistence agriculture and small-scale domesticated animal farming. There is a minimal presence of local businesses, and these are largely focused on obtaining products from the nearest city to resell locally at a much higher price, e.g. selling a minimal mobile top up voucher at more than $50 \%$ markup. The area is also home to a well-known surf spot, with a backpackers situated nearby. The beach is gorgeous, and the rocky reefs also attract fishermen and divers. These tourists stay at the backpackers that also serves as the headquarters for a local NGO (non-governmental organization) that actively tackles health, social, educational and economic needs in the community. Only a privileged few of the community residents have access to grid electricity. This power supply is unreliable and is out at least once a month. Repair teams from the city must negotiate bad and/or flooded roads during rain storms in the rainy season especially, and repairs can be delayed for days.

The nearest city to Mankosi, Umthatha, is situated $70 \mathrm{~km}$ away, of which $40 \mathrm{~km}$ are bad dirt roads over hilly terrain and river passes. This can take 1.5 hours to negotiate with a high clearance vehicle, and can take up to 4 hours with local transport used by community residents. One can find basic amenities in the city; for example food, tools and stationary are easily available, yet specialized electronic or telecommunications devices are nowhere to be found and need to be imported from bigger cities further afield, e.g. $200 \mathrm{~km}$ or more away. Despite the community's relative isolation (by South African standards), mobile phone coverage is widespread, albeit spotty in places requiring one to walk up this or that hill to obtain coverage. This year saw the introduction of $3 \mathrm{G}$ in one small corner of the community where a hotel and a number of tourist houses are located near the beach. According to a previous study by one of this paper's co-authors, $60 \%$ of people over 14 years of age own a mobile phone. However, as stated above, most community members do not have grid electricity domestically, and must walk to a power point and pay to charge a phone, e.g. a small shop, informal bar, a neighbour's solar panel or generator, or at the nearby backpackers. People pay in local currency an average of US\$0.65 to charge a phone [10]. GPRS and EDGE are as available as GSM, yet very few people can use it. Firstly, because most of their low-end phones do not support cellular data, and second, because even if they did, people could not af- ford the usual unbundled US $\$ 0.23 / \mathrm{MB}$ unless they became sophisticated Instant Messaging users. At the NGO's headquarters, there is a VSAT connected to a WiFi hotspot that provides Internet access to tourists (for a price, of course) and to NGO staff. Apart from the NGO's interventions in the community, several DEV initiatives have taken place near and within this particular community, by members of this team, e.g. rural wireless telehealth [31], mobile callback [10] and a mobile audio repository [24].

This mesh network project is following in the footsteps of those interventions. Given the airtime consumption pattern of mostly local cellular calls made within the community, a wireless mesh network to support 'free' voice over IP (VoIP) calls inside the community was considered. However, as free calls will essentially render the network financially unsustainable, it was decided by the TA to develop a business model to charge a low rate for calls to keep the mesh network up and running. The TA proposed charging for intra-community calls to enable the network to become financially sustainable, so that a local support team can make a living from maintaining, and even expanding, the network. Scenarios comprised of different usage and pricing patterns were explored in order to find the break-even point of the network [25]. At this stage, the pricing has not yet been implemented and will be driven by the TA.

\section{METHODOLOGY}

The community-driven mesh network rollout described in this paper has only been completed very recently. An initial visit of two weeks was made in April 2012 to explore feasibility of the idea with the TA, followed by a two month visit spanning June--July 2012, where training of the local support team, in their home language, enabled them to rollout an initial mesh deployment by themselves, including a custom solar power solution. From the very beginning of the project, it was decided that the research approach would follow on the methods culled from work related to our effort both internally and externally, i.e. to learn from others' experiences and design our initiative to adhere to documented best practice and avoid common pitfalls [11, 12, 14]. This section provides an overview of the main lessons taken from the literature, situated with how we have approached them together with our community. Given that the project currently aims to provide only internal VoIP calls, issues regarding subsequent Internet access and local content are not addressed herein, although we fully intend to address these and related issues at a later stage in the project's evolution.

\subsection{Overall approach}

Brewer et al. suggest that "ICT can enable new solutions only when applied with a broad understanding and a multidisciplinary approach" [11]. Our team is made of people with different backgrounds and approaches to DEV in rural areas, with cumulative experience gained in several countries. Furthermore, to reduce the inter-cultural gap, two co-authors of this paper speak the local language as a first language: one who is from a major city, speaks fluent English, is a postgraduate student in Computer Science and can help bridge communication gaps between researchers and locals in the community; and the other who is born in the local community and has lived there ever since, also speaks English, and more importantly, can help bridge cultural gaps between the community and the research team. 


\subsection{Local origination}

Bhatnagar [9] states that a DEV project must be originated locally, and "cannot be forced down the throats" of locals, in order to meet local needs. Other authors may not mention the origin of an idea, yet still stress the importance of meeting local needs as a key ingredient of the success of the project [11] and the long term commitment of a given community [23]. In our case, we first visited the community to assess the possibility of deploying a wireless mesh network there. We interviewed several people with semi-structured interviews, and with their answers, together with the results of our previous research in the area and our knowledge of the potential of the technology, we came to the idea of using the mesh nodes as public phones for providing cheaper calls within the community. Strictly speaking, the initial idea did not come from the community, although the final idea was informed by its members and their socio-technical environment.

Once we had a clear picture of the initial community mesh idea, we met with the Headman, as this is the protocol when anyone wants to start a project in the community. We described the intervention's pros and cons to him. As he considered the project beneficial to the community, he called a bigger meeting with his advisers, including sub-headmen, messengers, advisors, and other people interested in the development of the community. We presented the idea again to the larger TA, and after receiving positive feedback, we were granted permission to work in the community. This may not suffice for guaranteeing that projects like this "serve a public good" [11], e.g. perhaps in this case, because we consulted only the community TA, and only the ruling institution itself might benefit. This is a risk that we must take, and is discussed in detail in Section 4.3.

\subsection{Partnering with local institutions}

Partnering with local institutions, especially local governments and NGOs, is also considered very important[23], since they "tend to understand local needs and dynamics in a way that is not possible from afar" [11]. This may or may not be true across the board in our country, where, from our experience, many still adhere to 'old-school' development assumptions concerning the poor's needs without ever consulting them. Also, here it is often believed that local government representatives benefit only themselves. Regardless, we have established strong partnerships with a local NGO and the local government, the TA.

The commitments made by the technical research team, based at a major national university, were the delivery of a minimum number of solar powered nodes (eight, that ended up being ten) that would be installed for the mesh network to provide voice services throughout the community, and provision of training for their installation and management. The TA committed to choose the households for the mesh nodes according to a pre-discussed set of constraints (see Section 3.4) to ensure that each household receiving a node would not misuse the batteries, for example; and to decide the mechanisms and pricing to charge for the calls in order to achieve financially sustainability. We also expect that a TA-endorsed team will manage and operate the network, as in [23], and this will be discussed in more detail below. As part of the partnership with the local NGO, we have been allowed to install a data-collection server on a laptop on their premises, to record call detail records and quality of service (QoS) metrics, for free; and they also agreed to provide access to the NGO's tools and vehicles (for local transport) when needed for the project.

\subsection{Community co-design}

Community co-design to take advantage of local knowledge is also considered another important factor given every DEV intervention's "unique regional and cultural characteristics of the area" [11]. Co-design provides a sense of ownership to the beneficiaries concerning the project [9]. As stated above, the TA has been an active part of the design of the network, especially by proposing households that comply with the constraints decided among the different partners, following a similar approach to [23]:

- Meet the physical constraints required by the wireless mesh nodes, e.g. electromagnetic line of sight among nodes considering the Fresnel zone; link budget calculations to check that the received power over a certain distance is above the receiver sensitivity, with a maximum distance of 1 kilometer, a constraint given the radio characteristics of the mesh router chosen; and the concept of a mesh node, with each node having to "see" at least three others.

- Benefit the institutions working in the community.

- Benefit the community at large, i.e. the household selected being surrounded by other households.

- Provide security for the installation, to prevent theft of devices, specifically by having some kind of fence around the house or at least many people living in and around the homestead.

Given the hilly terrain where the community is located, the proposed node positioning provided by the community was if not the most optimum one, at least close to it. The locals are intimately familiar with poor GSM coverage in the area; that it is better on hilltops. It is worth noting that the TA's node location choices met the constraints aforementioned while simultaneously satisfying local politics, e.g. ten nodes are now located in ten of the twelve villages that make up the community (which has, incidentally, caused problems related in Section 4.4). If GPS coordinates and Radio Mobile were the only tools available to design the network, as some sources may point out [15], the installation team would have been surprised at the number of links that appear feasible in software that are actually not possible due to the existence of scattered houses and trees that can block links. These do not appear in the digital maps due to their low resolution. Thus, local knowledge played an essential role in designing the mesh topology.

As the network it is still in its infancy, several months of trials are needed to collect data before the TA can make an informed decision concerning how to charge for calls made. We have not yet worked with the TA on the business model, and it will be co-designed, too, or at least designed by them with some input from the research team. So far, they clearly understand that some form of income is needed to maintain the network in the future, because it is they who suggested charging for intranet calls in the first place. This is important because it denotes a shift away from 'old school' development work where the intervention remains externally funded until the funding runs out and the project eventually becomes abandoned. 


\subsection{Local champion}

The existence of a local champion for taking situated leadership on different aspects of the project is considered of great importance [9, 14]. Johnson and Roux even consider it the first factor for success [20], given the different rates of growth in the two projects they compared: one with a local champion and one without. In our case, the local-born co-author, currently paid by the lead university's research budget hand-in-hand with the local NGO, plays this role. $\mathrm{He}$ is a well respected young man who is well received by all stakeholders in the project. He also has several years of experience with ICT. In addition to the single champion idea, which is also susceptible to that person leaving the area, our approach is more aligned to that of community wireless networks where responsibility is shared out among different users of the network. We employ a network of Local Research Assistants (LRAs), based on success with this approach with previous projects $[10,24]$. We also do this in order to avoid local champion block-out, i.e. taking sole ownership of the project. In other words, if the local champion 'disappears' from or 'dictates' the project for any reason whatsoever, there will always be a back up. As such, the aforementioned co-author is also documenting the entire operation and maintenance of the mesh network, in the local language, specifically to ease handover should it be required in the future. However, the project is still in too early a stage for us to validate the results of this approach.

\subsection{Capacity building}

Many agree on the need to develop local competencies as key to a successful intervention [21, 20, 5, 13]. Given the lack of homegrown ICT talent in rural areas [27], many interventions are carried out by specialists from outside the community, who are expected to transfer the maintenance to local entrepreneurs [20] or to staff up from the local public administration [5].

The approach we followed was to build that capacity into the installation of the entire network, and all the other subsystems required, as part and parcel of the training. Thus, we proposed that the TA call for and appoint members of the community who were interested in the training sessions, provided that we offered them transportation and food during the proceedings. The response to the general call was encouraging and a team of $4-5$ people was waiting to be picked up every day over a span of two months, and this was without pay. In the initial training sessions, we covered the theory and then moved on to hands-on practical exercises, e.g. working with wood to construct custom boxes to hold the batteries, installing and dimensioning solar and storage systems, building the connection panel for the electrical installation, configuring and installing the routers, and aligning their antennae. However, once a first 'sample' was constructed by a trainer, e.g. a solar battery storage box or Mesh Potato pole mount, the trainees implemented additional units by themselves under the watchful eye of a trainer. Although this may sound relatively easy, it took much effort to apply in practice and often delayed the training schedule, given the time needed for problem solving and the time constraints of the research team in the field (only two months). We intended this approach of 'training by doing' to pass on and internalize concepts with hands-on skills; to increase confidence among the maintenance team. In addition, we aimed, by working in teams, to lead up to the concept of community wireless networks, where each piece of the system is key for the overall system to run smoothly. In subsequent discussions, trainees highlighted the benefits of team work over any other lesson learned, as this approach nicely dovetails with local traditions.

End user training is also considered very important to some authors, since local lack of knowledge is one of the reasons for network downtime [20,4]. Our approach here was not just explaining practical maintenance tasks to the users $^{1}$. We also provided more in-depth detail about how things work, especially the electrical power, for them to be able to make decisions consciously and independently. One member from each homestead, then, received such training once the electrical installation was completed. In that training session, the local champion explained, with the help of other people in the support team, concepts regarding security and consumption of battery power. In addition to this, to further improve the knowledge of potential users, an extra training session will be provided in each household, given by our first line of trainees. It is intended that this approach will improve the capacity of a wider group of end users when facing a problem, thus reducing the number of interventions from the local support team of technicians, and from them to us.

Both the installation and user training were given by locals. This was proposed in order to avoid the "only the outsider knows how to do things" with technology that is situated in the community. To take this approach further, we have already asked the local support team to replace antennae without us. Upon initial rollout, we were disappointed to find that the antennae we purchased did not have the gain attributed on its spec sheet. We had used the aforementioned Radio Mobile tool to verify that community-chosen node sites would work, especially regarding distances of up to $2 \mathrm{~km}$ between nodes. This did not go well in the field, as the antennae were not strong enough. Therefore, we ordered stronger antennae, and verified their output at our campus before having these antennae delivered to the community for the local team to change out without our help. We are hoping that these antennae will result in improved connectivity and QoS, and demonstrate that this 'hand-over' approach can be better than a traditional "let me help you" approach regarding local technicians' capacity to maintain the network after initial installation. We have therefore not installed remote monitoring or access on purpose, and are keen to encourage the local support team to take credit for 'fixing' the network.

It is worth again noting that both types of training were given in the local language. If the trainer were Englishspeaking, then a translation was done to the local language, and trainees also had the opportunity to use an interpreter to ask questions if they were not comfortable with English. Given that there are not yet that many server applications in the project, and that there is no access to the Internet from the mesh yet, we hope that with this effort we can account for the concerns of some, e.g. [11] regarding the use of local languages as far as user interfaces are concerned. Additionally, for the few administrative tools that are used, e.g.

\footnotetext{
${ }^{1}$ By user, we mean those having a mesh node and a charging system in the house, since we do not consider that the rest of the users, those who could potentially use the system in other people's houses, need any training to make a phone call.
} 
on the server, they are in English, and we are considering approaching a national translation team to provide native language support for the open source tools we want to use. However, we are not sure if that might also entail adapting the products to the local culture [9], and we hope this not going to be a major barrier to use of the management tools.

\subsection{Electrical Power}

The unreliability of grid electricity in deeply rural areas is frequently one of the main challenges of this type of project $[21,11,23]$. Grid electricity in our community is scarce, and its unreliability compounded by multi-day outages that occur monthly. To avoid reliance on mains power, all mesh public phones are powered via deep cycle $12 \mathrm{v}$ batteries, mostly charged by solar panels. With this solution we also address environmental concerns raised by some, e.g. [11], since although the batteries used are not environmentally friendly, other solutions, such as a generator, are much more polluting. Note that in our particular community, we are loath to use wind turbines, despite the prevalence of strong wind throughout the year, due to the noise generated. We have had prior experience with wind causing grid antennae to vibrate loudly causing locals to believe that their ancestors were communicating with them. So we placed plastic strips in the antennae to dampen that sound, and are now sensitive to creating any form of noise pollution.

Technical and budgetary details of the solar systems installed in eight homesteads can be found elsewhere [25]. The systems were intentionally over-dimensioned to allow users to charge mobile phones, their own and those of other community members, as part of the business model, and to allow users to plug other devices such as a light and/or radio, to foster a sense of ownership with the project and thus encourage care of the system. There are currently three nodes powered by an off-the-self battery solution with smaller capacity that provides electricity solely for a given node. For one of these, we opted to charge the battery with what we know is unreliable mains power, although the mesh node still runs off of the battery. This imported off-the-shelf system has unfortunately proven to be incompatible with rural needs, as after four days of rain and cloud, the solar charged system simply went down. The problem would not be so important if it were not necessary to press a restart button every time that happens. We also noted that the voltage meter is constantly on; on our solution, we press a doorbell button to inspect the voltage, thus removing a constant drain, albeit tiny, on the battery. We need to better understand the impact of these issues before considering substituting these power solutions.

The only node that is not powered independently is the monitoring server and a mesh node attached to it. These are behind a battery array at the backpackers that allow for some nominal number of hours of independence. During the installation we realized that if a power outage is longer than 24 hours, the server goes down. A solution must be found for this, if we want to collect data reliably.

\subsection{Robust technology}

The choice of the device to form a mesh network is also an important factor toward overall success. Providing that the device complies with the technology one wants to use, in this case $\mathrm{WiFi}$ mesh, there are several factors to consider, including low cost [27], ease of use [3] and maintainability
[5]. Furthermore, since it is difficult in developing countries like ours to find such items locally at low cost, we are forced to purchase items abroad and suffer the consequences of local transportation, where things can easily get damaged $[20,12]$. Once installed, devices can also suffer from harsh environments that may destroy them [3, 20, 12].

We have chosen to use the 'Mesh Potato' [32] in order to try to tackle most of the challenges mentioned above. Firstly, the device is low cost at US\$79 or lower depending on quantity purchased, and consumes low power (0.3A). Secondly, it comes with an integrated Analog Telephone Adapter (ATA) that allows for the provision of telephony without adding an extra device that would increase the power consumption. The ATA port is a standard RJ11 that takes a standard PSTN, or POTS, handset. The Mesh Potato has been designed for harsh environments and is available online, with several notable deployments already existing, lending credence to its robustness. In addition, the operating system and the user interface have been designed for non-technical users, and it is fairly straightforward to create a mesh and make calls 'out of the box'. We can also 'play around' with the open source protocols and applications if we so desire.

In order to combat the harsh environment where our network is located, mainly due to sand, wind, and rain, additional effort has been made to buy most of auxiliary materials rust-proof, and protect those that are not with primer. To handle the wind, poles were fixed to the roof in an umbrella-like shape to limit movement, and an inventive constraint also attaches an antenna to the pole.

Nevertheless, we have fallen into one of the pitfalls mentioned in related work: the "inconsistent performance of lowcost hardware" [3]. When the TA gave us the location of the nodes, we realized that the network could be made much more robust by increasing the number of links, and making the existing ones stronger, if external antennae were added to the nodes. We did the handiwork to add the external antenna to the Mesh Potato only to realize that the antennae (a supposedly $9 \mathrm{dBi}$ dipole antenna, designed for indoors) actually only performed as a directional one. We managed to get end-to-end connectivity by having one link at a time, but not enough to allow for end-to-end calls. As described above in Section 3.6, tried and tested outdoor antennae have recently been shipped to the community for the local support team to replace the originals.

\subsection{Maintenance, support, and learning}

Once a network is installed, its maintenance over time is key to keeping it up and running [5]. To provide it efficiently [5] proposed an 'Operative Maintenance Plan' consisting of five levels of maintenance. We have condensed these into only three levels. While our Level 0 is similar, and we expect users to carry out basic maintenance tasks, Levels 1 and 2 from [5], consisting of local technicians and more educated technicians from the nearest city, respectively, are merged into our Level 1, since we have trained local technicians to possess the confidence necessary to address problems by themselves. Levels 3 and 4 from [5], where high level support and research activities for improving services are carried out, respectively, are combined to form our Level 2, that is expected to be carried out by the Village Telco community, where several of the authors are active members. It is through this online community that a more on-demand 
distance learning mentioned by some, e.g. [20, 5], will take place in our project; although only for the researchers and local technicians, and not for the users. For improving maintenance of the network, a management server, Nagios, and a server for visualizing active links and nodes, SPUD, have been installed on the laptop server mentioned above. In addition, to facilitate maintenance, the electrical installation has been designed and constructed in a modular way, allowing each piece of the system to be disconnected for repair independently, e.g. a panel with a user console on top and the wiring beneath, and a separate breathable area to house the batteries. The rationale behind the box design has been explained to the trainees in depth, including how to change fuses and replace them by bigger ones, and the consequences of doing so; again to encourage end users to learn about solar and electricity basics, to allow them to gain confidence while at the same time facilitating localized maintenance.

\subsection{Institutional and financial sustainability}

Financial sustainability of a given project is one of the main concerns in the DEV literature [7, 4, 11]. In this project, the revenue generated from the calls made in the network are expected to cover the running expenses and even generate revenue for scaling the network, or can even be used for other projects that the community prioritizes. We have run preliminary costing exercises that identify possible break-even points based on various costing and usage scenarios. However, income generation does not necessarily guarantee sustainability. In the DEV literature, some also stress the importance of institutional sustainability, in order for a given network to continue providing services initially envisioned once a project is left to the receiving institution to manage $[23,4,9,7]$. Some propose the creation of a local NGO to do so [23], and others advocate creating departments and/or roles needed, in existing institutions to take a project further [4]. Indeed there is consensus on the need for a smooth transfer of leadership to such a local institution. In our case, such a transition is not even necessary, since the network already belongs de facto to the community, with the TA, via weekly community meetings, already taking all of the administrative decisions. The network is planned to be managed, once operational, by this paper's co-author resident in the community, although his remuneration will eventually depend on the revenue generated by the system. In addition, the TA is taking steps to have a local 'trust' manage the money emanating from the 'goods' of the community, to manage the financial aspects of the network and handle money collection and dispersement, thus relieving sole responsibility of our resident 'champion'.

\section{DISCUSSION OF CULTURAL FACTORS}

Having covered the factors highlighted in the literature and how we are addressing such concerns with this particular project, we now turn to discuss the local cultural factors that we are discovering as more time is spent in the community; factors that significantly influence the deployment of the wireless mesh network with and for the community.

\subsection{Time}

Any community owned initiative takes considerable time in negotiating and resolving issues, and this is particularly true in our community. Firstly, the TA considers community participation in meetings to be a very important part of governing and maintaining cohesion. Secondly, actually co-ordinating and getting to meetings takes considerable time. Issues that might seem rather small within other socio-technical systems are rather large in a community that emphasizes "working together"; where the scarcity of all resources makes them particularly valuable and also envied if people feel that some are privileged over others. Coordinating meetings requires contacting community members by phone, which may not be charged for up to several days, due to lack of grid electricity. Alternatively, talking in person can often involve walking great distances. Thus attending meetings can involve hours of travel. We find ourselves balancing the economics that constrain our time, e.g. project funding and expertise availability, with the time necessary for genuine local ownership and decision-making.

However, if we shift focus away from implementing a system, or solving social issues according to a research schedule, and within an external time-frame, we realize that local practices around time are critically important to sustaining communication systems. Practices in close knit rural African communities configure time as polychronic, including being based around people, rather than based on a limited abstraction of hours or days. Investing time in conversation and resolving social issues provides the community with its resilience and is framed within social relationships that extend across life-times and social networks that extend over centuries. That is, the value of time spent communicating for a close knit rural community differs from the values that emerge in societies that have many weak and dynamic social ties. The social cost of an uninformed, controversial or opaque decision about the system is extremely high. For instance, the TA will endure criticism for many years if residents feel that they were not appropriately consulted or decisions were biased, and this will have consequences for their relationships within the community. Thus, at least for rural Africa, we propose that sustained success in $\mathrm{WiFi}$ deployments involves conceptualizing project time scales in local terms.

Building upon relationships and the understandings established during an 18 month communication project that ran prior to this mesh project provided a highly facilitative context. In that project, the researcher lived locally, and took a participative, ethnographic approach. Residents gained considerable experience in addressing technical and socio-technical challenges that arose over a prolonged period of time. This enabled residents to approach the current project in a more informed way. For instance, the beginning of the project coincided with the TA facing the issue of sabotage of one of the stations from the previous project. That station had been sabotaged by family members in the homestead the station had been placed, at least in part, because the TA and local community members, especially women, somewhat enforced the station upon them. It should be noted, too, that the prior project also followed on precedents established by earlier projects, demonstrating the benefit of having successive projects within a single longitudinal arc.

\subsection{Gender and age}

In prior projects, we learned that women's access was better if women were involved in management. Thus we sought to train both men and women for this project, and the TA also nominated the home of a woman who performs subheadman's duties. However, in this traditional social ecosystem, 
a woman's responsibilities make it difficult for her to sustain long-term attendance at training; in our case, almost two months. Three women appeared in the very first sessions of the training. However, while they appeared enthusiastic and engaged, only one could make it to later stages of the training, and with much less frequency than men. In one earlier project in the area, we were able to sustain women's engagement because they controlled their hours of work, which they organized around their domestic and child-care responsibilities, in co-ordination with other women. This suggests that greater flexibility in training, such as shorter sessions at more convenient locations, and a more extended training period might have enabled women to participate more consistently.

We asked the TA to propose people suited to training, and these people, in most cases, were much younger than those whose homes the phones are deployed. This reflects their recognition that younger people are considerably more technologically literate and, at least from their elders' perspective, have more time to spend on this project. However, it also means that those trained are also less likely to be able to enforce rules since the community has a very strong elder authority protocol. We hope that as a group, those trained will act together to supervise use and mitigate against use of the voice services by the TA at the exclusion of other community members. However, we are very aware that although younger people in the community tend to be critical of the TA, they are also reserved in voicing critique and may be shy when visiting an elder's home.

\subsection{Governance and trust}

We chose to work with the local TA because it represents the one single structure that exists within and connects across all villages within the community, and convenes weekly meetings within that community. Our prolonged immersion, living in the community in an earlier project, suggest that the TA pursues consensus in decision making and the community has recognized that the TA attempted to ensure that the infrastructure was available to all. However, as in all communities, perspectives about who is privileged by decisions differ. So, it is common that people assume the Headman is amenable to a small token to, say, put forward a person for a job, or assign one of the mesh nodes to a particular homestead. We are cautious to generalize cases in the literature where leaders influence projects in ways that do not align with community needs. Certainly, we proceed carefully in assuming the TA can enable broad community 'involvement' and access, and still argue that understanding the meaning of 'fairness' within a close-knit rural African community that does not prioritize democracy over consensus, emerges by engaging with the local governing structures.

There is considerable trust within this community compared with many places we have personally visited in our country, which suggests the potential for achieving access to the stations by the community. People have extensive kin relations within and across the villages, and since they spend much of life outdoors, they are visible to and familiar with each other. Homes are often left unlocked, though small shops and established informal bars have grilled doors and barbed wire fences. Some people have reputations for pilfering, including from family members although, generally, theft locally is spoken about as committed by, or in collaboration with, criminals from beyond the village. Inhabitants reiterated that the "community must work together" on matters of theft; and, indeed, we retrieved a phone stolen in a previous project, buried in bushes out of view, by agreeing not to disclose the thief's identity or report him to the police or the TA.

\subsection{Interpreting practice}

We are only now beginning to comprehend the social and cross-cultural complexities that emerge in implementing, with and for a close-knit rural community, an idea conceived by people external to the community. We sought to produce local ownership and stimulate enterprise by framing local engagement in training and decision-making as voluntary rather than as a job paid for by an external research project. This demands the community to take on responsibility in ways that are, at best, unfamiliar and at worse uncomfortable for them. Attributing problems to outsiders, such as provincial departments, NGOs, experts or 'Western' people, is one way in which the community avoids internal conflict and maintains internal cohesion. Thus, we have found it difficult in both this and previous projects to progress from local expectations that we, as experts, will solve all problems and fix all breakages. Politicians in our country continue to gain power through promises of work, homes and pensions for those who were previously marginalized. The dominant narrative in our current society continues to blame all social inequality on the former regime. At the same time residents privilege visible conformance so individual enterprise can incur high social penalty. Conformist values in close-knit societies where people depend on others for their survival and psychological wellness are extremely powerful. It is all too easy to interpret, for instance, local reluctance to introduce and commit to responsibility enterprises as a "lack of entrepreneurial spirit", without accounting for the social penalties. We cannot assume that residents will necessarily perceive volunteers who are trained or homesteads in which stations are placed act in cooperative community interests. Indeed, community members must manage the jealousy of others with whom they live when budget-constrained projects are introduced to the community. Although we navigated through this with complete transparency in every step we have taken so far, discussing every aspect with the TA, for them to communicate or deal with the community, the aforementioned reasons may create bigger problems than the solutions are expected to provide due to this intracommunity jealousy. So far, the members of one of the two villages where there is no public phone has declined to fill out questionnaires for baseline data until they get a public phone there, although requested to help with the data collection by the Headman. Something similar happened with the last public phone to be allocated, where members from one of the villages came to the Headman's house to claim it, since they were told it was going to be allocated to a Headman's adviser in a village that already had one. The other village that does not have a public phone did not receive one either, not only for the budget constraints, but also as a punishment by the TA for another intra-community issue. Thus, the introduction of the mesh nodes has already created tensions within the community politics, or boosted those already existing, while at the same time can provide negotiating tools to relax them. 


\subsection{Respect and transparency}

The approach we followed in this project is based on the premise of providing the community with the knowledge and experience for keeping the network running without the continuous intervention of people from outside the community. Thus, we have acted with sincere respect to local traditions. In addition, for achieving real emancipation and a sense of ownership, additional steps need to be taken. For example, considering the community the true owner of the network, with us having to ask for permission for modifying something, obtaining data or even showing it to our peers. In other words, we do not consider the network as ours ${ }^{2}$. This respect is based on considering the locals as important as ourselves in this mutually beneficial arrangement; acknowledging and making clear that the community is providing as many benefits to us, and not only in research terms, as we are providing to them. Sometimes in research, just by giving something, researchers expect to be able to get any data they want from an isolated and impoverished community, without even explaining why the data is important and without considering what people would do just to receive something shiny, new and exciting, like a public Mesh Potato. Alongside this, we have also encountered situations in this initial rollout where people expected equipment just because they were providing data to us. By acknowledging a mutual equality, and engaging in discussions where everyone has the chance to understand the reasoning behind choices made, more progress can be made. Breaking these barriers takes time on both sides, especially given the existing power structures and racial clashes that still predominate in this country. We fully acknowledge that it is going to be difficult to assess whether this approach is more successful than others, and in our opinion, is still worth trying.

\section{CONCLUSION AND FUTURE WORK}

In this paper, we have reflected on the efforts made to rollout an inclusive and participatory community co-designed mesh network to provide internal voice calls within Mankosi, a rural impoverished community. We have tried to realize the initiative following state-of-the-art research, and by considering both technical and non-technical recommendations found in the literature in order to follow best practice and avoid pitfalls of DEV projects. In doing so, we have come to appreciate the complexity of culture in the social and physical context where the network is located, and its importance when deploying a wireless mesh network, something that we feel is missing in the literature consulted. Although we are continuing to improve our understanding of that complexity, we have already pinpointed significant cultural factors that may influence the success of the deployment, and we described how we have addressed them. Having only just completed the initial rollout, it is presumptuous on our part to make any claim on sustainability at this stage, although we expect that the methodology that we have followed will lead us to both achieve mutual goals for the mesh network and also to better understand the factors that lead us toward that achievement together with the community. We feel such lessons are valuable for other members of the DEV community.

During the next stages of the project we will study dif-

\footnotetext{
${ }^{2}$ This leads to another concern that we paid for the initial
} rollout with research funding. ferent calling scenarios, given that unlike the mobile phones that locals are accustomed to, the operator of the receiving public phone will pick up a shared mesh phone on the other end, without knowing the identity of the desired receiver for the call, who may or may not be present a the other end. These scenarios will influence the pricing model(s) that the TA comes up with, and can strongly influence the usage of the system. Based on that emergent model, we must then design a billing system that allows for the different possibilities and is adaptable to the local culture in order to provide trustworthy transparency to the operation and usage of the network. Furthermore, other issues like privacy, opening hours of the public phones, and socio-economic impact of the network will be analyzed as we collect data from the usage of the network. Alongside such data, we are also measuring performance terms of QoS, availability and usage with software tools like CDRStats and Nagios (with MRTG) that have been installed and configured as part of the initial rollout. We have initiated a baseline study with questionnaires administered by local research assistants, among 257 households. We recently received some questionnaires from the field, and are anxious to being examining the baseline data, that will later be compared against follow-up versions of the questionnaires. Such methods will be combined with more narrative ones in order to triangulate quantitative and qualitative data to better understand the impact of the project. Depending on the demand and the success of the business model scaling up network, the provision of additional services like breakout calls, access to the Internet and adding tablets and smartphones seamlessly with a WiFi interface to make calls in the network might also be considered. However, all future efforts will be driven by, with and for the community.

\section{ACKNOWLEDGMENTS}

The authors would like to thank members of Mankosi community and Transcape for their support of the daily activities in the field. This work has been funded by Telkom SA, Cisco SA, Aria Technologies SA and the THRIP programme at the South African Department of Trade and Industry (the dst) via the Telkom Centre of Excellence (CoE) programme. THRIP funding is managed by the National Research Foundation (NRF), and the NRF also provided additional funding. Any opinion, findings and conclusions or recommendations expressed in this material are those of the authors and therefore the NRF does not accept any liability in regard thereto. Additional funding was provided by the Shuttleworth Foundation.

\section{REFERENCES}

[1] AirJaldi Wireless Network. drupal.airjaldi.com/.

[2] A. Anand, V. Pejovic, D. L. Johnson, and E. M. Belding. Villagecell: cost effective cellular connectivity in rural areas. In Proc. 5th IEEE/ACM International Conference on ICTD, pages 180-189, Georgia Tech, Atlanta, GA, 2012.

[3] J. Backens, G. Mweemba, and G. van Stam. A Rural Implementation of a 52 node mixed Wireless Mesh Network in Macha, Zambia. In Africomm '09, 2009.

[4] I. Bebea-González, L. Linán, and C. Rey-Moreno. Design of a Sustainability Action Plan for EHAS-Napo project: a rural e-Health initiative. In Proc. 4 th 
IEEE/ACM International Conference on ICTD, Royal Holloway, London, United Kingdom, 2010.

[5] I. Bebea-González, J. Paco, L. Linán, J. Simó-Reigadas, and A. Martínez-Fernández. Management Framework for Sustainable e-Healthcare Provision. In Proc. IADIS International Conference e-Society, Avila, Spain, 2011.

[6] M. Best. The Wireless Revolution and Universal Access. Trends in Telecommunications Reform, pages 107-122, 2003.

[7] M. Best and R. Kumar. Sustainability Failures of Rural Telecenters: Challenges from the Sustainable Access in Rural India (SARI) Project. Information Technologies and International Development, 4(4):31-45, 2008.

[8] P. Bhagwat, B. Raman, and D. Sanghi. Turning 802.11 Inside-Out. ACM SIGCOMM Computer Communication Review, 34(1):33-38, January 2004.

[9] S. Bhatnagar. Social Implications of Information and Communication Technology in Developing Countries: Lessons from Asian Success Stories. Electronic Journal on Information Systems in Developing Countries, 1(4):1-9, 2000.

[10] N. J. Bidwell, M. Lalma, G. Marsden, B. Dlutu, S. Ntlangano, A. Manjingolo, W. D. Tucker, M. Jones, R. Robinson, E. Vartianen, and I. Klanpanos. Please call ME.N.U.4EVER: Designing for 'Callback' in Rural Africa. In IWIPS'11 (10th Intl. Workshop on Internationalisation of Products and Systems), pages 117-138, 2011.

[11] E. Brewer, M. Demmer, B. Du, M. Ho, M. Kam, S. Nedevschi, J. Pal, R. Patra, S. Surana, and K. Fall. The Case for Technology in Developing Regions. IEEE Computer, 38(6):25-38, 2005.

[12] E. Brewer, M. Demmer, M. Ho, R. J. Honicky, J. Pal, M. Plauché, and S. Surana. The Challenges of Technology Research for Developing Regions. Pervasive Computing, 5(2):15-23, 2006.

[13] Bridges.org. Real Access / Real Impact criteria. http://www.bridges.org, 2004.

[14] Bridges.org. 12 habits of highly effective ICT-enabled development initiatives. http://www.bridges.org/12_habits, 2005.

[15] R. Flickenger, C. Aichele, C. Fonda, J. Forster, I. Howard, T. Krag, and M. Zennaro. Wireless Networking in the Developing World. Limehouse Book Sprint Team, 2006.

[16] M. Foth. The Handbook of Research on Urban Informatics: The Practice and Promise of Real-Time City. Idea Group Inc (IGI), 2009.

[17] Fundación EHAS. http://www.ehas.org.

[18] H. Galperin. Wireless Networks and Rural Development: Opportunities for Latin America. Information Technologies and International Development, 2(3):47-56, 2005.

[19] J. Hausman. Mobile Phones in Developing Countries. In New Goods and Improvements in Living Standards, 2010 Cornucopia Quantified, 2010.

[20] D. Johnson and K. Roux. Building Rural Wireless Networks: Lessons Learnt and Future Directions. In WiNS-DR'08, pages 17-22, 2008.
[21] K. Mathee, G. Mweemba, A. Pais, G. V. Stam, and M. Rijken. Bringing Internet Connectivity to Rural Zambia Using a Collaborative Approach. In Proc. 2nd IEEE/ACM International Conference on ICTD, pages 1-12, 2007.

[22] Nepal Wireless. nepalwireless.net.

[23] A. Nungu, T. Brown, and B. Pehrson. Challenges in Sustaining Municipal Broadband Networks in the Developing World. In ICeND 2011, pages 24-40, 2011.

[24] T. Reitmaier, N. J. Bidwell, J. S. Masbulele, W. D. Tucker, and G. Marsden. Communicating in designing an oral repository for rural African villages. In Information Society Technologies - Africa (IST-Africa). IIMC International Information Management Corporation, 2012.

[25] C. Rey-Moreno, Z. Roro, M. J. Siya, J. Simo-Reigadas, N. J. Bidwell, and W. D. Tucker. Towards a Sustainable Business Model for Rural Telephony. In Proc. of the III International Workshop on Research on ICT for Human Development, 2012.

[26] C. Rey-Moreno, J. Simo-Reigadas, and A. Martinez-Fernandez. Wimax: An Uncertain Promise for Bridging the Digital Divide. IEEE Technology and Society Magazine, 30(4):26-33, 2011.

[27] J. Simo-Reigadas, A. Martinez, P. Osuna, S. Lafuente, and J. Seoane. The Design of a Wireless

Solar-Powered Router for Rural Environments Isolated from Health Facilities. IEEE Wireless Communications, 15(3):24-30, June 2008.

[28] S. Song. 3 New Village Telco Entrepreneur Profiles. http://villagetelco.org/2012/08/3-new-village-telcoentrepreneur-profiles/.

[29] L. Subramanian, S. Surana, R. Patra, S. Nedevschi, M. Ho, E. Brewer, and A. Sheth. Rethinking Wireless in the Developing World. Hot Topics in Networks, 2006.

[30] W. D. Tucker and E. H. Blake. Abstractions for Designing and Evaluating Communication Bridges for People in Developing Regions. In First ACM Symposium on Computing for Development (ACM DEV), 2010.

[31] W. D. Tucker, E. H. Blake, G. Marsden, M. Pearson, and R. Westerveld. Reflection on three years of rural wireless Internet Protocol communication. In D. Browne, editor, Southern African

Telecommunication Networks and Applications Conference (SATNAC), pages 452-457. Telkom, 2007.

[32] Village Telco. villagetelco.org.

[33] R. Westerveld. Inverse Telecommunications: The Future for Rural Areas in Developing Countries? In T. M. Egyedi and D. C. Mehos, editors, Inverse Infrastructures: Disrupting Networks from Below, pages 187-207. Edward Elgar Publishing Ltd, 2011. 